\title{
On bivariate Meyer-König and Zeller operators
}

\author{
Ali Olgun
}




\title{
ON BIVARIATE MEYER-KÖNIG AND ZELLER OPERATORS
}

\author{
ALI OLGUN
}

Received 3 May, 2012

\begin{abstract}
This work relates to bivariate Meyer-König and Zeller operators, $M_{n}, n \in \mathbb{N}$ which are not a tensor product setting. We show the monotonicity of the sequence of operators for $n$ under convexity, moreover we study the property of monotonicity in the sense of Li [9]. Finally, we provide an $r t h$ order generalization $M_{n}^{[r]}$ of $M_{n}$ and also study approximation of $M_{n}^{[r]}$.

2010 Mathematics Subject Classification: 41A25; 41A36
\end{abstract}

Keywords: multivariate Meyer-König and Zeller operator, convexity, monotonicity, modulus of continuity

\section{INTRODUCTION}

The Cheney and Sharma modification of the well-known univariate Meyer-König and Zeller operators (MKZ) are defined as

$$
M_{n}^{*}(f, x)=\sum_{k=0}^{\infty} m_{n, k}(x) f\left(\frac{k}{n+k}\right), n \in \mathbb{N}
$$

for $f \in C[0,1)$ and $x \in[0,1), n \in \mathbb{N}$, where $m_{n, k}(x)=(1-x)^{n+1}\left(\begin{array}{c}n+k \\ k\end{array}\right) x^{k}[3]$.

The monotonic convergence of $\left\{M_{n}^{*}(f ; x)\right\}_{n=1}^{\infty}$ under convexity was investigated in [2] by Cheney and Sharma by means of analytical approach, also studied in [8] by Khan, by means of probabilistic approach. Monotonic convergence of several approximation operators are studied deeply in [6] by Khan, Della-Vecchia and Fassih with probabilistic point of view. Some works related to MKZ operators can be viewed in $[1-8,13]$ and $[10,11]$.

We shall use the following standard notation. Let $\mathbf{x}=\left(x_{1}, x_{2}\right), \mathbf{t}=\left(t_{1}, t_{2}\right) \in \mathbb{R}^{2}$, $\mathbf{k}=\left(k_{1}, k_{2}\right) \in \mathbb{N}_{0}^{2}, \mathbb{N}_{0}=\mathbb{N} \cup\{0\} . \mathbf{x}^{\mathbf{k}}=x_{1}^{k_{1}} \cdot x_{2}^{k_{2}}, \mathbf{k} !=k_{1} ! \cdot k_{2} !,|\mathbf{k}|=k_{1}+k_{2},|\mathbf{x}|=$ 
$x_{1}+x_{2}, \mathbf{e}_{i}$ denotes the unit vector in $\mathbb{R}^{2}$. Furthermore $\left(\begin{array}{l}n \\ \mathbf{k}\end{array}\right)=\frac{n !}{\mathbf{k} !(n-|\mathbf{k}|) !}$, and $\sum_{\mathbf{k}=0}^{\infty}=\sum_{k_{1}=0}^{\infty} \sum_{k_{2}=0}^{\infty}$

Let $S_{2} \subset \mathbb{R}^{2}$ be the open simplex defined by

$$
S_{2}=\left\{\mathbf{x} \in \mathbb{R}^{2} ; \mathbf{x}_{i} \geq 0, i=1,2,|\mathbf{x}|<1\right\} .
$$

For a function $f \in C\left(S_{2}\right)$, bivariate $\mathrm{MKZ}$ operators are defined as

$$
M_{n}(f, \mathbf{x})=(1-|\mathbf{x}|)^{n+1} \sum_{\mathbf{k}=0}^{\infty} f\left(\frac{\mathbf{k}}{n+|\mathbf{k}|}\right)\left(\begin{array}{c}
n+|\mathbf{k}| \\
\mathbf{k}
\end{array}\right) \mathbf{x}^{\mathbf{k}}, n \in \mathbb{N},
$$

where $C\left(S_{2}\right)$ denotes the space of continuous real valued functions defined on $S_{2}$. It is obvious that $M_{n}, n \in \mathbb{N}$, are not a tensor product extension of the univariate MKZ operators $M_{n}^{*}$ given by (1.2).

Now we give the following definitions which we shall use.

Definition 1. A continuous function $f$ is said to be convex in $D \subset \mathbb{R}^{m}$, if

$$
f\left(\sum_{i=1}^{r} \alpha_{i} \mathbf{x}_{i}\right) \geq \sum_{i=1}^{r} \alpha_{i} f\left(\mathbf{x}_{i}\right)
$$

for every $x_{1}, x_{2}, \ldots, x_{r} \in D$ and for every non-negative numbers $\alpha_{1}, \alpha_{2}, \ldots, \alpha_{r}$ such that $\alpha_{1}+\alpha_{2}+\ldots+\alpha_{r}=1$.

Definition 2. A continuous function $f$ from $D \subseteq \mathbb{R}^{2}$ into $\mathbb{R}$ is said to be Lipschitz continuous of order $\mu, \mu \in(0,1]$, if there exists a constant $A>0$ such that for every $\left(x_{1}, x_{2}\right),\left(y_{1}, y_{2}\right) \in D, f$ satisfies

$$
\left|f\left(x_{1}, x_{2}\right)-f\left(y_{1}, y_{2}\right)\right| \leq A \sum_{i=1}^{2}\left|x_{i}-y_{i}\right|^{\mu},
$$

the set of Lipschitz continuous functions is denoted by $\operatorname{Lip}_{A}(\mu, D)$.

In this work, we firstly show the monotonicity of the sequence of bivariate MKZ operators defined by (1.2) under convexity. Secondly we give a kind of monotonnicity similar to the property given by $\mathrm{Li}$ in [9]. Namely, we show that if $f(\mathbf{x})$ is a nonnegative function and $x_{i}^{-1} f(\mathbf{x})(i=1,2)$ is non-increasing for $x_{i}$ on $(0,1)$, then for each $n \geq 1, x_{i}^{-1} M_{n}(f ; \mathbf{x})$ is also non-increasing for $x_{i}$ on $(0,1)$. Moreover we build an $r-t h$ order generalization $M_{n}^{[r]}$ of $M_{n}$ analogues to Kirov and Popova's construction in [8] and investigate its approximation property. 


\section{Monotonicity for the Sequence of Bivariate Meyer-König And ZELLER OPERATORS}

In this section, we study the monotonic convergence of the sequence of bivariate MKZ operator under convexity. Note that monotonic convergence of univariate MKZ operator when $f$ is convex, was first obtained by Cheney and Sharma in [3]. We note here that monotonic convergence of the multivariate Baskakov operator is studied in [2].

Theorem 1. If $f$ is convex, then $M_{n}(f ; \mathbf{x})$ is strictly monotonically non-decreasing in $n$, unless $f$ is the linear function (in which case $M_{n}(f ; \mathbf{x})=M_{n+1}(f ; \mathbf{x})$ for all $n \in \mathbb{N})$.

Proof. We have

$$
\begin{aligned}
& M_{n}(f ; \mathbf{x})-M_{n+1}(f ; \mathbf{x})=(1-|\mathbf{x}|)^{n+1} \times \\
& \times\left\{\sum_{k_{2}=1}^{\infty}\left[f\left(0, \frac{k_{2}}{n+k_{2}}\right)\left(\begin{array}{c}
n+k_{2} \\
k_{2}
\end{array}\right)-f\left(0, \frac{k_{2}}{n+1+k_{2}}\right)\left(\begin{array}{c}
n+1+k_{2} \\
k_{2}
\end{array}\right)\right] \mathbf{x}^{\mathbf{k}}\right. \\
& +\sum_{k_{1}=1}^{\infty}\left[f\left(\frac{k_{1}}{n+k_{1}}, 0\right)\left(\begin{array}{c}
n+k_{1} \\
k_{1}
\end{array}\right)-f\left(\frac{k_{1}}{n+1+k_{1}}, 0\right)\left(\begin{array}{c}
n+1+k_{1} \\
k_{1}
\end{array}\right)\right] \mathbf{x}^{\mathbf{k}} \\
& +\sum_{k_{1}=0}^{\infty} \sum_{k_{2}=0}^{\infty} f\left(\frac{\mathbf{k}}{n+1+|\mathbf{k}|}\right)\left(\begin{array}{c}
n+1+|\mathbf{k}| \\
\mathbf{k}
\end{array}\right) x_{1}^{k_{1}+1} x_{2}^{k_{2}} \\
& \left.+\sum_{k_{1}=0}^{\infty} \sum_{k_{2}=0}^{\infty} f\left(\frac{\mathbf{k}}{n+1+|\mathbf{k}|}\right)\left(\begin{array}{c}
n+1+|\mathbf{k}| \\
\mathbf{k}
\end{array}\right) x_{1}^{k_{1}} x_{2}^{k_{2}+1}\right\} \text {. }
\end{aligned}
$$

Therefore the last equation reduces to the following:

$$
\begin{aligned}
& M_{n}(f ; \mathbf{x})-M_{n+1}(f ; \mathbf{x})=(1-|\mathbf{x}|)^{n+1} \times \\
& \times\left\{\sum _ { k _ { 2 } = 1 } ^ { \infty } \left[f\left(0, \frac{k_{2}}{n+k_{2}}\right)\left(\begin{array}{c}
n+k_{2} \\
k_{2}
\end{array}\right)-f\left(0, \frac{k_{2}}{n+1+k_{2}}\right)\left(\begin{array}{c}
n+1+k_{2} \\
k_{2}
\end{array}\right)\right.\right. \\
& \left.+f\left(0, \frac{k_{2}-1}{n+k_{2}}\right)\left(\begin{array}{c}
n+k_{2} \\
k_{2}-1
\end{array}\right)\right] x_{2}^{k_{2}}+\sum_{k_{1}=1}^{\infty}\left[f\left(\frac{k_{1}}{n+k_{1}}, 0\right)\left(\begin{array}{c}
n+k_{1} \\
k_{1}
\end{array}\right)\right. \\
& \left.-f\left(\frac{k_{1}}{n+1+k_{1}}, 0\right)\left(\begin{array}{c}
n+1+k_{1} \\
k_{1}
\end{array}\right)+f\left(\frac{k_{1}-1}{n+k_{1}}, 0\right)\left(\begin{array}{c}
n+k_{1} \\
k_{1}-1
\end{array}\right)\right] x_{1}^{k_{1}} \\
& +\sum_{k_{1}=1}^{\infty} \sum_{k_{2}=1}^{\infty}\left[f\left(\frac{k_{1}-1}{n+|\mathbf{k}|}, \frac{k_{2}}{n+|\mathbf{k}|}\right)\left(\begin{array}{c}
n+|\mathbf{k}| \\
\mathbf{k}-e_{1}
\end{array}\right)\right.
\end{aligned}
$$




$$
\begin{aligned}
& +f\left(\frac{k_{1}}{n+|\mathbf{k}|}, \frac{k_{2}-1}{n+|\mathbf{k}|}\right)\left(\begin{array}{c}
n+|\mathbf{k}| \\
\mathbf{k}-e_{2}
\end{array}\right) \\
& \left.\left.+f\left(\frac{\mathbf{k}}{n+|\mathbf{k}|}\right)\left(\begin{array}{c}
n+|\mathbf{k}| \\
\mathbf{k}
\end{array}\right)-f\left(\frac{\mathbf{k}}{n+1+|\mathbf{k}|}\right)\left(\begin{array}{c}
n+1+|\mathbf{k}| \\
\mathbf{k}
\end{array}\right)\right] \mathbf{x}^{\mathbf{k}}\right\} .
\end{aligned}
$$

Let $I_{1}, I_{2}$ and $I_{3}$ denote the followings

$$
\begin{aligned}
& I_{1}=f\left(\frac{\mathbf{k}}{n+|\mathbf{k}|}\right)\left(\begin{array}{c}
n+|\mathbf{k}| \\
\mathbf{k}
\end{array}\right)-f\left(\frac{\mathbf{k}}{n+1+|\mathbf{k}|}\right)\left(\begin{array}{c}
n+1+|\mathbf{k}| \\
\mathbf{k}
\end{array}\right) \\
&+f\left(\frac{\mathbf{k}-\mathbf{e}_{1}}{n+|\mathbf{k}|}\right)\left(\begin{array}{c}
n+|\mathbf{k}| \\
\mathbf{k}-\mathbf{e}_{1}
\end{array}\right)+f\left(\frac{\mathbf{k}-\mathbf{e}_{2}}{n+|\mathbf{k}|}\right)\left(\begin{array}{c}
n+|\mathbf{k}| \\
\mathbf{k}-\mathbf{e}_{2}
\end{array}\right), \\
& I_{2}=f\left(0, \frac{k_{2}}{n+k_{2}}\right)\left(\begin{array}{c}
n+k_{2} \\
k_{2}
\end{array}\right)-f\left(0, \frac{k_{2}}{n+1+k_{2}}\right)\left(\begin{array}{c}
n+1+k_{2} \\
k_{2}
\end{array}\right) \\
&+f\left(0, \frac{k_{2}-1}{n+k_{2}}\right)\left(\begin{array}{c}
n+k_{2} \\
k_{2}-1
\end{array}\right), \\
& I_{3} f\left(\frac{k_{1}}{n+k_{1}}, 0\right)\left(\begin{array}{c}
n+k_{1} \\
k_{1}
\end{array}\right)-f\left(\frac{k_{1}}{n+1+k_{1}}, 0\right)\left(\begin{array}{c}
n+1+k_{1} \\
k_{1}
\end{array}\right) \\
&+
\end{aligned}
$$

For $I_{1}$, we take

$$
\alpha_{1}=\frac{\left(\begin{array}{c}
n+|\mathbf{k}| \\
\mathbf{k}
\end{array}\right)}{\left(\begin{array}{c}
n+1+|\mathbf{k}| \\
\mathbf{k}
\end{array}\right)}, \alpha_{2}=\frac{\left(\begin{array}{c}
n+|\mathbf{k}| \\
\mathbf{k}-\mathbf{e}_{1}
\end{array}\right)}{\left(\begin{array}{c}
n+1+|\mathbf{k}| \\
\mathbf{k}
\end{array}\right)}, \alpha_{3}=\frac{\left(\begin{array}{c}
n+|\mathbf{k}| \\
\mathbf{k}-\mathbf{e}_{2}
\end{array}\right)}{\left(\begin{array}{c}
n+1+|\mathbf{k}| \\
\mathbf{k}
\end{array}\right)} .
$$

Clearly $\alpha_{1}, \alpha_{2}$ and $\alpha_{3}$ are non-negative numbers, and $\alpha_{1}+\alpha_{2}+\alpha_{3}=1$. On the other hand if we set

$$
\mathbf{x}_{1}=\frac{\mathbf{k}}{n+|\mathbf{k}|}, \quad \mathbf{x}_{2}=\frac{\mathbf{k}-\mathbf{e}_{1}}{n+|\mathbf{k}|}, \quad \mathbf{x}_{3}=\frac{\mathbf{k}-\mathbf{e}_{2}}{n+|\mathbf{k}|},
$$

then it follows that

$$
\alpha_{1} \mathbf{x}_{1}+\alpha_{2} \mathbf{x}_{2}+\alpha_{3} \mathbf{x}_{3}=\frac{\mathbf{k}}{n+1+|\mathbf{k}|}
$$


Hence, from the convexity of $f$ we obtain that $I_{1} \leq 0$. For $I_{2}$, we set

$$
\alpha_{1}=\frac{\left(\begin{array}{c}
n+k_{2} \\
k_{2}
\end{array}\right)}{\left(\begin{array}{c}
n+1+k_{2} \\
k_{2}
\end{array}\right)}, \alpha_{2}=\frac{\left(\begin{array}{c}
n+k_{2} \\
k_{2}-1
\end{array}\right)}{\left(\begin{array}{c}
n+1+k_{2} \\
k_{2}
\end{array}\right)}
$$

and

$$
\mathbf{y}_{1}=\left(0, \frac{k_{2}}{n+k_{2}}\right), \mathbf{y}_{2}=\left(0, \frac{k_{2}-1}{n+k_{2}}\right),
$$

then, clearly, $\alpha_{1}, \alpha_{2} \geq 0$, and $\alpha_{1}+\alpha_{2}=1$. Morever we have

$$
\alpha_{1} \mathbf{y}_{1}+\alpha_{2} \mathbf{y}_{2}=\left(0, \frac{k_{2}}{n+k_{2}}\right)
$$

Thus, from the convexity of $f$ we obtain that $I_{2} \leq 0$. Similarly we deduce that $I_{3} \leq$ 0 . So we have proved $M_{n}(f ; \mathbf{x}) \leq M_{n+1}(f ; \mathbf{x})$ for all $n \in \mathbb{N}$. Using the similar arguments given in [2], the last part of the proof is given as follows: The equality of $M_{n}(f ; \mathbf{x})$ and $M_{n+1}(f ; \mathbf{x})$ can be satisfied only if $I_{1}=0$ for all $k_{1}, k_{2} \in \mathbb{N}$ and $I_{2}=I_{3}=0$ for all $k_{1}, k_{2} \in \mathbb{N}_{0}$. But, if $f$ is convex, then $I_{1}=0$ implies that its graph is a plane in triangle.

In the following, we show that the bivariate MKZ operators can retain a certain monotony, that is

Theorem 2. Let $f$ be defined on $S_{2}$. If $f(\mathbf{x})$ is a non-negative function such that $\frac{f(\mathbf{x})}{x_{i}}(i=1,2)$ is non-increasing for $x_{i}$ on $(0,1)$, then for each $n \geq 1, \frac{M_{n}(f ; \mathbf{x})}{x_{i}}$, is also non-increasing for $x_{i}$ on $(0,1)$.

Proof. Straightforward computation gives that for $n \geq 1$ we have, for example with respect to $x_{1}$,

$$
\begin{aligned}
& \frac{\partial}{\partial x_{1}}\left(\frac{M_{n}(f ; \mathbf{x})}{x_{1}}\right)= \\
& =\frac{\partial}{\partial x_{1}}\left\{\sum_{k_{1}=0}^{\infty} \sum_{k_{2}=0}^{\infty} \frac{\left(1-x_{1}-x_{2}\right)^{n+1}}{x_{1}} f\left(\frac{k_{1}}{n+k_{1}+k_{2}}, \frac{k_{2}}{n+k_{1}+k_{2}}\right)\left(\begin{array}{c}
n+k_{1}+k_{2} \\
k_{1}+k_{2}
\end{array}\right) x_{1}^{k_{1}} x_{2}^{k_{2}}\right\} \\
& =\frac{\partial}{\partial x_{1}}\left\{\sum_{k_{2}=0}^{\infty} \frac{\left(1-x_{1}-x_{2}\right)^{n+1}}{x_{1}} f\left(0, \frac{k_{2}}{n+k_{2}}\right)\left(\begin{array}{c}
n+k_{2} \\
k_{2}
\end{array}\right) x_{2}^{k_{2}}+\sum_{k_{1}=1}^{\infty} \sum_{k_{2}=0}^{\infty}\right. \\
& \left.\times\left(1-x_{1}-x_{2}\right)^{n+1} f\left(\frac{k_{1}}{n+k_{1}+k_{2}}, \frac{k_{2}}{n+k_{1}+k_{2}}\right)\left(\begin{array}{c}
n+k_{1}+k_{2} \\
k_{1}+k_{2}
\end{array}\right) x_{1}^{k_{1}-1} x_{2}^{k_{2}}\right\} \\
& =\sum_{k_{2}=0}^{\infty} \frac{\partial}{\partial x_{1}}\left(\frac{\left(1-x_{1}-x_{2}\right)^{n+1}}{x_{1}}\right) f\left(0, \frac{k_{2}}{n+k_{2}}\right)\left(\begin{array}{c}
n+k_{2} \\
k_{2}
\end{array}\right) x_{2}^{k_{2}}+\sum_{k_{1}=1}^{\infty} \sum_{k_{2}=0}^{\infty}
\end{aligned}
$$




$$
\begin{aligned}
& \times f\left(\frac{k_{1}}{n+k_{1}+k_{2}}, \frac{k_{2}}{n+k_{1}+k_{2}}\right)\left(\begin{array}{c}
n+k_{1}+k_{2} \\
k_{1}+k_{2}
\end{array}\right) \frac{\partial}{\partial x_{1}}\left[\left(1-x_{1}-x_{2}\right)^{n+1} x_{1}^{k_{1}-1} x_{2}^{k_{2}}\right] \\
& =\sum_{k_{2}=0}^{\infty}\left(\frac{-(n+1)\left(1-x_{1}-x_{2}\right)^{n} x_{1}-\left(1-x_{1}-x_{2}\right)^{n+1}}{x_{1}^{2}}\right) f\left(0, \frac{k_{2}}{n+k_{2}}\right)\left(\begin{array}{c}
n+k_{2} \\
k_{2}
\end{array}\right) x_{2}^{k_{2}} \\
& +\sum_{k_{1}=1}^{\infty} \sum_{k_{2}=0}^{\infty} f\left(\frac{k_{1}}{n+k_{1}+k_{2}}, \frac{k_{2}}{n+k_{1}+k_{2}}\right)\left(\begin{array}{c}
n+k_{1}+k_{2} \\
k_{1}+k_{2}
\end{array}\right) x_{2}^{k_{2}} \\
& \times\left[-(n+1)\left(1-x_{1}-x_{2}\right)^{n} x_{1}^{k_{1}-1}+\left(1-x_{1}-x_{2}\right)^{n+1}\left(k_{1}-1\right) x_{1}^{k_{1}-2}\right] \\
& =\sum_{k_{2}=0}^{\infty} \frac{\left(1-x_{1}-x_{2}\right)^{n+1}}{x_{1}^{2}}\left(\frac{-(n+1) x_{1}}{1-x_{1}-x_{2}}-1\right) f\left(0, \frac{k_{2}}{n+k_{2}}\right)\left(\begin{array}{c}
n+k_{2} \\
k_{2}
\end{array}\right) x_{2}^{k_{2}} \\
& +\sum_{k_{1}=1}^{\infty} \sum_{k_{2}=0}^{\infty} f\left(\frac{k_{1}}{n+k_{1}+k_{2}}, \frac{k_{2}}{n+k_{1}+k_{2}}\right)\left(\begin{array}{c}
n+k_{1}+k_{2} \\
k_{1}+k_{2}
\end{array}\right)\left(1-x_{1}-x_{2}\right)^{n} x_{2}^{k_{2}} \\
& \times\left[-n x_{1}^{k_{1}-1}-x_{1}^{k_{1}-1}+\left(k_{1}-1\right) x_{1}^{k_{1}-2}-\left(k_{1}-1\right) x_{1}^{k_{1}-1}-\left(k_{1}-1\right) x_{1}^{k_{1}-2} x_{2}\right] \\
& \times x_{1}^{k_{1}-2}\left(1-x_{2}\right)\left(1-x_{1}-x_{2}\right)^{n} x_{2}^{k_{2}} \\
& =\sum_{k_{2}=0}^{\infty} \frac{\left(1-x_{1}-x_{2}\right)^{n+1}}{x_{1}^{2}}\left(-\frac{\left(1+n x_{1}-x_{2}\right)}{1-x_{1}-x_{2}}\right) f\left(0, \frac{k_{2}}{n+k_{2}}\right)\left(\begin{array}{c}
n+k_{2} \\
k_{2}
\end{array}\right) x_{2}^{k_{2}} \\
& +\sum_{k_{1}=1}^{\infty} \sum_{k_{2}=0}^{\infty} f\left(\frac{k_{1}}{n+k_{1}+k_{2}}, \frac{k_{2}}{n+k_{1}+k_{2}}\right)\left(\begin{array}{c}
n+k_{1}+k_{2} \\
k_{1}+k_{2}
\end{array}\right)\left(1-x_{1}-x_{2}\right)^{n} x_{2}^{k_{2}} \\
& \times\left[-\left(n+k_{1}\right) x_{1}^{k_{1}-1}+\left(k_{1}-1\right)\left(1-x_{2}\right) x_{1}^{k_{1}-2}\right] \\
& =\sum_{k_{2}=0}^{\infty} \frac{\left(1-x_{1}-x_{2}\right)^{n}}{x_{1}^{2}}\left(-\left(1+n x_{1}-x_{2}\right)\right) f\left(0, \frac{k_{2}}{n+k_{2}}\right)\left(\begin{array}{c}
n+k_{2} \\
k_{2}
\end{array}\right) x_{2}^{k_{2}} \\
& +\sum_{k_{1}=1}^{\infty} \sum_{k_{2}=0}^{\infty} f\left(\frac{k_{1}}{n+k_{1}+k_{2}}, \frac{k_{2}}{n+k_{1}+k_{2}}\right)\left(\begin{array}{c}
n+k_{1}+k_{2} \\
k_{1}+k_{2}
\end{array}\right) x_{1}^{k_{1}-1}\left(-n-k_{1}\right) x_{2}^{k_{2}} \\
& \times\left(1-x_{1}-x_{2}\right)^{n}+\sum_{k_{1}=2}^{\infty} \sum_{k_{2}=0}^{\infty} f\left(\frac{k_{1}}{n+k_{1}+k_{2}}, \frac{k_{2}}{n+k_{1}+k_{2}}\right)\left(\begin{array}{c}
n+k_{1}+k_{2} \\
k_{1}+k_{2}
\end{array}\right) \\
& =\sum_{k_{2}=0}^{\infty} \frac{-\left(1+n x_{1}-x_{2}\right)}{x_{1}^{2}} f\left(0, \frac{k_{2}}{n+k_{2}}\right)\left(\begin{array}{c}
n+k_{2} \\
k_{2}
\end{array}\right)\left(1-x_{1}-x_{2}\right)^{n} x_{2}^{k_{2}} \\
& -\sum_{k_{1}=1}^{\infty} \sum_{k_{2}=0}^{\infty} f\left(\frac{k_{1}}{n+k_{1}+k_{2}}, \frac{k_{2}}{n+k_{1}+k_{2}}\right)\left(\begin{array}{c}
n+k_{1}+k_{2} \\
k_{1}+k_{2}
\end{array}\right) x_{1}^{k_{1}-1}\left(n+k_{1}\right) x_{2}^{k_{2}}
\end{aligned}
$$




$$
\begin{aligned}
& \times\left(1-x_{1}-x_{2}\right)^{n}+\sum_{k_{1}=1}^{\infty} \sum_{k_{2}=0}^{\infty} f\left(\frac{k_{1}+1}{n+k_{1}+k_{2}}, \frac{k_{2}}{n+k_{1}+k_{2}}\right)\left(\begin{array}{c}
n+k_{1}+1+k_{2} \\
k_{1}+1+k_{2}
\end{array}\right) \\
& \times\left(1-x_{2}\right) x_{1}^{k_{1}-1} k_{1}\left(1-x_{1}-x_{2}\right)^{n} x_{2}^{k_{2}} \\
& \leq-\sum_{k_{2}=0}^{\infty} \frac{\left(1+n x_{1}-x_{2}\right)}{x_{1}^{2}} f\left(0, \frac{k_{2}}{n+k_{2}}\right)\left(\begin{array}{c}
n+k_{2} \\
k_{2}
\end{array}\right)\left(1-x_{1}-x_{2}\right)^{n} x_{2}^{k_{2}} \\
& +\sum_{k_{1}=1}^{\infty} \sum_{k_{2}=0}^{\infty} f\left(\frac{k_{1}+1}{n+k_{1}+k_{2}+1}, \frac{k_{2}}{n+k_{1}+k_{2}+1}\right)\left(\begin{array}{c}
n+k_{1}+k_{2} \\
k_{1}+k_{2}
\end{array}\right) \frac{n+k_{1}+k_{2}+1}{k_{1}+k_{2}+1} \frac{k_{1}}{x_{1}} \\
& \times\left(1-x_{2}\right)\left(1-x_{1}-x_{2}\right)^{n} x_{1}^{k_{1}} x_{2}^{k_{2}}-\sum_{k_{1}=1}^{\infty} \sum_{k_{2}=0}^{\infty} f\left(\frac{k_{1}}{n+k_{1}+k_{2}}, \frac{k_{2}}{n+k_{1}+k_{2}}\right)\left(\begin{array}{c}
n+k_{1}+k_{2} \\
k_{1}+k_{2}
\end{array}\right) \\
& \times \frac{\left(n+k_{1}\right)}{k_{1}} \frac{k_{1}}{x_{1}}\left(1-x_{1}-x_{2}\right)^{n} x_{1}^{k_{1}} x_{2}^{k_{2}}
\end{aligned}
$$

Since for $k_{1}, k_{2} \geq 0$

$$
\frac{n+k_{1}+k_{2}+1}{k_{1}+k_{2}+1}<\frac{n+k_{1}+k_{2}+1}{k_{1}+1}, \quad-\frac{n+k_{1}+k_{2}}{k_{1}}<-\frac{n+k_{1}}{k_{1}}
$$

and

$$
1+n x_{1}-x_{2} \geq 0,1-x_{2} \leq 1, \text { for } x_{1}, x_{2} \in S_{2}, n \in \mathbb{N},
$$

so we have for $n \geq 1$

$$
\begin{aligned}
& \frac{\partial}{\partial x_{1}}\left(\frac{M_{n}(f ; \mathbf{x})}{x_{1}}\right) \leq \\
& \leq-\sum_{k_{2}=0}^{\infty} \frac{\left(1+n x_{1}-x_{2}\right)}{x_{1}^{2}} f\left(0, \frac{k_{2}}{n+k_{2}}\right)\left(\begin{array}{c}
n+k_{2} \\
k_{2}
\end{array}\right)\left(1-x_{1}-x_{2}\right)^{n} x_{2}^{k_{2}} \\
& \quad+\sum_{k_{1}=1}^{\infty} \sum_{k_{2}=0}^{\infty}\left\{f\left(\frac{k_{1}+1}{n+k_{1}+k_{2}+1}, \frac{k_{2}}{n+k_{1}+k_{2}+1}\right) \frac{n+k_{1}+k_{2}+1}{k_{1}+k_{2}+1}\right. \\
& \left.\quad-f\left(\frac{k_{1}}{n+k_{1}+k_{2}}, \frac{k_{2}}{n+k_{1}+k_{2}}\right) \frac{\left(n+k_{1}+k_{2}\right)}{k_{1}}\right\} \\
& \quad \times\left(\begin{array}{c}
n+k_{1}+k_{2} \\
k_{1}+k_{2}
\end{array}\right) \frac{k_{1}}{x_{1}}\left(1-x_{1}-x_{2}\right)^{n} x_{1}^{k_{1}} x_{2}^{k_{2}},
\end{aligned}
$$

is non-positive, since $f$ is a non-negative function, such that $\frac{f(\mathbf{x})}{x_{i}}(i=1,2)$ is nonincreasing for $x_{i}$ on $(0,1)$. Similar calculations can be obtained for $x_{2}$. 


\section{A Generalization}

This section provides an $r$-th order generalization of the bivariate MKZ operators in the sense of Kirov and Popova's construction [8].

Let $C^{r}\left(S_{2}\right), r \in \mathbb{N}_{0}$, denote the space of all functions $f$ defined on $S_{2}$ and having all continuous partial derivatives up to order $r$. By $M_{n}^{[r]}$, we denote the following generalization of $M_{n}$. For $\mathbf{x}, \mathbf{t} \in S_{2}$,

$$
M_{n}^{[r]}(f ; \mathbf{x})=M_{n}\left(P_{r, \frac{\mathbf{k}}{n+|\mathbf{k}|}}(\Delta \mathbf{x}, f)\right),
$$

where

$$
\begin{aligned}
\Delta \mathbf{x}:=\left(\Delta x_{1}, \Delta x_{2}\right) & =\mathbf{x}-\frac{\mathbf{k}}{n+|\mathbf{k}|}=\left(x_{1}-\frac{k_{1}}{n+|\mathbf{k}|}, x_{2}-\frac{k_{2}}{n+|\mathbf{k}|}\right), \\
\nabla & =\left(\frac{\partial}{\partial x_{1}}, \frac{\partial}{\partial x_{2}}\right), \\
(\Delta \mathbf{x} . \nabla)^{r} & :=\sum_{i+j=r}\left(\begin{array}{l}
r \\
j
\end{array}\right)\left(\Delta x_{1}\right)^{i}\left(\Delta x_{2}\right)^{j} \frac{\partial^{r}}{\partial x_{1}^{i} \partial x_{2}^{j}},
\end{aligned}
$$

$\left(\begin{array}{l}r \\ j\end{array}\right)$ are binomial coefficients, and

$$
\begin{aligned}
P_{r, \frac{\mathbf{k}}{n+|\mathbf{k}|}}(\Delta \mathbf{x}, f) & =f\left(\frac{\mathbf{k}}{n+|\mathbf{k}|}\right)+(\Delta \mathbf{x} \cdot \nabla) f\left(\frac{\mathbf{k}}{n+|\mathbf{k}|}\right) \\
& +\frac{(\Delta \mathbf{x} \cdot \nabla)^{2}}{2 !} f\left(\frac{\mathbf{k}}{n+|\mathbf{k}|}\right)+\ldots+\frac{(\Delta \mathbf{x} \cdot \nabla)^{r}}{r !} f\left(\frac{\mathbf{k}}{n+|\mathbf{k}|}\right),
\end{aligned}
$$

the Taylor polynomial for $f$ at $\frac{\mathbf{k}}{n+|\mathbf{k}|} \in S_{2}$.

Now we state the following pointwise estimate for $M_{n}^{[r]}$

Theorem 3. If $f \in C^{r}\left(S_{2}\right)$ and $\frac{\partial^{r} f}{\partial x_{1}^{i} \partial x_{2}^{j}} \in \operatorname{Li} p_{A}(\gamma), i+j=r$, then we have

$$
\left|M_{n}^{[r]}(f ; \mathbf{x})-f(\mathbf{x})\right| \leq \frac{2 A}{(r-1) !} \frac{\gamma}{\gamma+r} B(\gamma, r) M_{n}(g ; \mathbf{x})
$$

for $x \in S_{2}$, where $g(\mathbf{s})=|\mathbf{x}-\mathbf{s}|^{r+\gamma}, B(\gamma, r)$ is the familiar beta function, $r, n \in \mathbb{N}_{0}$, $0<\gamma \leq 1$ and $A>0$.

Proof. From (3.1) and (3.3) we have

$$
f(\mathbf{x})-M_{n}^{[r]}(f ; \mathbf{x})=\sum_{\mathbf{k}=0}^{\infty} R_{r, \frac{\mathbf{k}}{n+|\mathbf{k}|}}(\Delta \mathbf{x}, f)\left(\begin{array}{c}
n+|\mathbf{k}| \\
\mathbf{k}
\end{array}\right) \mathbf{x}^{\mathbf{k}}(1-|\mathbf{x}|)^{n+1},
$$


where

$$
R_{r, \frac{\mathbf{k}}{n+|\mathbf{k}|}}(\Delta \mathbf{x}, f):=f(\mathbf{x})-\sum_{h=0}^{r} \frac{(\Delta \mathbf{x} \cdot \nabla)^{h}}{h !} f\left(\frac{\mathbf{k}}{n+|\mathbf{k}|}\right)\left(\begin{array}{c}
n+|\mathbf{k}| \\
\mathbf{k}
\end{array}\right) \mathbf{x}^{\mathbf{k}}(1-|\mathbf{x}|)^{n+1} .
$$

(3.6) can be given by

$$
\begin{aligned}
R_{r, \frac{\mathbf{k}}{n+|\mathbf{k}|}}(\Delta \mathbf{x}, f) & =\frac{1}{(r-1) !} \int_{0}^{1}(\Delta \mathbf{x} \cdot \nabla)^{r} \\
& \times\left[f\left(\frac{\mathbf{k}}{n+|\mathbf{k}|}+t \Delta \mathbf{x}\right)-f\left(\frac{\mathbf{k}}{n+|\mathbf{k}|}\right)\right](1-t)^{r-1} d t .
\end{aligned}
$$

Using (3.2),(3.7) results in

$$
\begin{aligned}
R_{r, \frac{\mathbf{k}}{n+|\mathbf{k}|}}(\Delta \mathbf{x}, f) & =\frac{1}{(r-1) !} \int_{0}^{1} \sum_{i+j=r}\left(\begin{array}{l}
r \\
j
\end{array}\right)\left(\Delta x_{1}\right)^{i}\left(\Delta x_{2}\right)^{j} \frac{\partial^{r}}{\partial x_{1}^{i} \partial x_{2}^{j}} \\
& \times\left[f\left(\frac{\mathbf{k}}{n+|\mathbf{k}|}+t \Delta \mathbf{x}\right)-f\left(\frac{\mathbf{k}}{n+|\mathbf{k}|}\right)\right](1-t)^{r-1} d t .
\end{aligned}
$$

Substituting (3.8) into (3.5) and taking into account that $\frac{\partial^{r} f}{\partial x_{1}^{i} \partial x_{2}^{j}} \in \operatorname{Li} p_{A}(\gamma)$ we arrive at the following.

$$
\begin{aligned}
\left|f(\mathbf{x})-M_{n}^{[r]}(f ; \mathbf{x})\right| & \leq \frac{A}{(r-1) !} \sum_{\mathbf{k}=0}^{\infty}\left(\left|\Delta x_{1}\right|+\left|\Delta x_{2}\right|\right)^{r}\left(\left|\Delta x_{1}\right|^{\gamma}+\left|\Delta x_{2}\right|^{\gamma}\right) \\
& \times\left(\begin{array}{c}
n+|\mathbf{k}| \\
\mathbf{k}
\end{array}\right) \mathbf{x}^{\mathbf{k}}(1-|\mathbf{x}|)^{n+1} \int_{0}^{1} t^{\gamma}(1-t)^{r-1} d t \\
& \leq \frac{2 A}{(r-1) !} \sum_{\mathbf{k}=0}^{\infty}\left(\left|\Delta x_{1}\right|+\left|\Delta x_{2}\right|\right)^{r+\gamma}\left(\begin{array}{c}
n+|\mathbf{k}| \\
\mathbf{k}
\end{array}\right) \mathbf{x}^{\mathbf{k}}(1-|\mathbf{x}|)^{n+1} B(\gamma+1, r) \\
& \leq \frac{2 A}{(r-1) !} \frac{\gamma}{\gamma+r} B(\gamma, r) \sum_{\mathbf{k}=0}^{\infty}\left|\mathbf{x}-\frac{\mathbf{k}}{n+|\mathbf{k}|}\right|^{r+\gamma}\left(\begin{array}{c}
n+|\mathbf{k}| \\
\mathbf{k}
\end{array}\right) \mathbf{x}^{\mathbf{k}}(1-|\mathbf{x}|)^{n+1},
\end{aligned}
$$

which proves (3.4).

For the uniform convergence of $M_{n}^{[r]}(f)$, let us consider the above mentioned function $g(\mathbf{s})=|\mathbf{x}-\mathbf{s}|^{r+\gamma}$. Obviously $g(\mathbf{x})=0$ and $g$ is continuous on $S_{2}$. From multivariate extension of the Bohman-Korovkin theorem (see [12]) we have

$$
\left\|M_{n}(g)\right\|_{C\left(S_{2}\right)} \rightarrow 0 \text { as } n \rightarrow \infty .
$$


Therefore by means of Theorem 3 we arrive at the following result

$$
\left\|M_{n}^{[r]}(f)-f\right\|_{C\left(S_{2}\right)} \rightarrow 0 \text { as } n \rightarrow \infty \text {. }
$$

\section{REFERENCES}

[1] A. Altın, O. Doğru, and F. Taşdelen, "The generalization of Meyer-König and Zeller operators by generating functions," J. Math. Anal. Appl., vol. 312, no. 1, pp. 181-194, 2005.

[2] F. Cao, C. Ding, and Z. Xu, "On multivariate Baskakov operator," J. Math. Anal. Appl., vol. 307, no. 1, pp. 274-291, 2005.

[3] E. W. Cheney and A. Sharma, "Bernstein power series," Can. J. Math., vol. 16, pp. 241-252, 1964.

[4] O. Doğru and O. Duman, "Statistical approximation of Meyer-König and Zeller operators based on $q$-integers," Publ. Math., vol. 68, no. 1-2, pp. 199-214, 2006.

[5] O. Doğru and V. Gupta, "Korovkin-type approximation properties of bivariate $q$-Meyer-König and Zeller operators," Calcolo, vol. 43, no. 1, pp. 51-63, 2006.

[6] M. Khan, B. Della Vecchia, and A. Fassih, "On the monotonicity of positive linear operators," $J$. Approx. Theory, vol. 92, no. 1, pp. 22-37, art. no. at963 113, 1998.

[7] R. A. Khan, "Some probabilistic methods in the theory of approximation operators," Acta Math. Acad. Sci. Hung., vol. 35, pp. 193-203, 1980.

[8] G. Kirov and L. Popova, "A generalization of the linear positive operators," Math. Balk., New Ser., vol. 7, no. 2, pp. 149-162, 1993.

[9] Z. Li, "Bernstein polynomials and modulus of continuity," J. Approx. Theory, vol. 102, no. 1, pp. 171-174, art. no. jath.1999.3374, 2000.

[10] A.-J. López-Moreno and F.-J. Muñoz-Delgado, "Asymptotic expansion of multivariate conservative linear operators," J. Comput. Appl. Math., vol. 150, no. 2, pp. 219-251, 2003.

[11] T. Trif, "An elementary proof of the preservation of Lipschitz constants by the Meyer-König and Zeller operators," JIPAM, J. Inequal. Pure Appl. Math., vol. 4, no. 5, p. 3, 2003.

[12] V. I. Volkov, "On the convergence of sequences of linear positive operators in the space of continuous functions of two variables," Dokl. Akad. Nauk SSSR, vol. 115, pp. 17-19, 1957.

[13] H. Wang, "Properties of convergence for the q-Meyer-König and Zeller operators," J. Math. Anal. Appl., vol. 335, no. 2, pp. 1360-1373, 2007.

\section{Author's address}

\section{Ali Olgun}

Kırıkkale University, Faculty of Art and Science, Department of Mathematics, 71450, Yahşihan, Kirıkkale, Turkey

E-mail address: aliolgun71@gmail.com 